\title{
Déterminants socio-économiques de l'utilisation des engins et pratiques de pêche destructives des ressources halieutiques sur le lac Toho au Bénin (Afrique de l'Ouest)
}

\author{
Victor $_{\text {CODJO }}{ }^{*}$, Afio ZANNOU ${ }^{2}$ et Gauthier BIAOU ${ }^{1,2}$ \\ ${ }^{1}$ Université Nationale d'Agriculture (UNA) du Bénin. \\ ${ }^{2}$ Laboratoire d'Economie Rurale et de Gestion des Exploitations Agricoles, Université d'Abomey Calavi \\ (UAC) au Bénin. \\ "Auteur correspondant ; E-mail : codjovictor@gmail.com; Tél. (+229) 96492185, \\ O2 BP 2110 Recette Principale, Cotonou 1. République du Bénin.
}

\section{RÉSUMÉ}

La pêche est une activité importante qui contribue à la sécurité alimentaire dans le monde entier, en particulier dans les pays en développement. Elle représente l'une des activités principales dans le septième Pôle de Développement Agricole au Bénin. Elle constitue pour les populations locales non seulement une source de protéines animales hautement nutritives mais aussi une activité génératrice de revenus. Elle est l'une des principales activités humaines qui affectent les écosystèmes aquatiques. L'objectif de cette étude était d'analyser les déterminants socio-économiques de l'utilisation des engins et pratiques de pêche destructives des ressources halieutiques. L'étude a porté sur 129 pêcheurs dans les villages Vèha et Logbo (commune de Lokossa) et Tokpa et Tohonou (commune de Houéyogbé). La méthode d'échantillonnage aléatoire simple a été utilisée pour la sélection des unités d'enquête. Un modèle Logit binomial a été utilisé pour l'analyse des déterminants socioéconomiques. Les résultats de l'étude ont montré que le nombre d'années d'expériences dans la pratique de la pêche, le niveau d'instruction du pêcheur et la fréquence de pêche sont les déterminants de l'utilisation des engins et pratiques de pêche destructives des ressources halieutiques au niveau du lac Toho. L'utilisation des filets réglementés, le développement d'activités génératrices de revenus autres que la pêche, la réduction de la pêche à la main, la réduction de l'utilisation des épuisettes et le développement de la pisciculture sont des stratégies de gestion développées par les pêcheurs face à la baisse des ressources halieutiques dans le lac Toho. (C) 2020 International Formulae Group. All rights reserved.

Mots clés : Ressources halieutiques, déterminants, engins et pratiques de pêche, modèle logit binomial.

\section{Socio-economic determinants of the use of destructive fishing gear and practices of fishery resources on Lake Toho in Benin (West Africa)}

\begin{abstract}
Fishery is an important activity contributing to food security in the world and particularly in developing countries. In south of Benin, fishery is one of the main activities in coastal regions. Fishery is not only a source of protein with high nutritious value but also an income generating activity. It is one of the main human activities which affect aquatic ecosystem. This study aims at analyzing the socio-economic determinants of the use of the destroy engine and practical in fisher. A total of 129 fishermen were interviewed in Vèha and Logbo villages in
\end{abstract}


the district of Lokossa and Tokpa and Tohonou villages in the district of Houéyogbé in South of Benin. A simple random sampling was used for selecting the survey units. Binomial Logit model was used to analyze the data. The results revealed that the number of years in fishery activity, the level of education and the frequency of fish harvesting are the socioeconomic determinants of the use of destroy practical in fishery in Toho lake. The use of regulated nets, the development of income-generating activities other than fishing, the reduction of catching fish by hand, the development of aquaculture and the development of the ritual practices, are ways to cope with the decline in halieutic resources of the fishermen.

(C) 2020 International Formulae Group. All rights reserved.

Keywords: Fishery resources, determinants, fishing gear and practices, Binomial Logit model.

\section{INTRODUCTION}

L'augmentation de la population mondiale a entraîné corrélativement une plus grande demande en protéines, notamment animales. $\mathrm{Au}$ niveau halieutique, l'amélioration des techniques de pêche, l'extension des zones de capture conduisant à accroître les captures ont permis de satisfaire une partie de cette demande protéique (FAO, 2014) entraînant un doublement des captures totales entre les années 1962 (40 000 tonnes) et 1987 (80 000 tonnes) (Roche International, 2000). La pêche est la seule source de protéines animales dans certains pays en voie de développement (Ticheler 2000) notamment au Bénin (UEMOA, 2002). Au Bénin, elle contribue pour $75 \%$ à la production halieutique nationale et participe pour près de $40 \%$ à la consommation nationale de protéines animales (UEMOA, 2002). En 2006, la production halieutique nationale est de l'ordre de 40000 tonnes dont $75 \%$ provenant de la pêche artisanale continentale. La pêche au Bénin est pratiquée par environ 40000 pêcheurs professionnels auxquels s'ajoutent 13000 saisonniers (MAEP, 2009).

La production halieutique au Bénin est jusqu'à ce jour essentiellement fournie par la pêche dans les cours et plans d'eau. Depuis quelques années, les besoins en poissons des populations sont accrus alors que les prises ont chuté passant de 41507 tonnes en 2006 à 36385 tonnes en 2007 (MAEP, 2009). Cette situation rend très dépendantes les populations locales vis-à-vis des ressources halieutiques. La pêche étant l'une des principales activités humaines qui affectent les écosystèmes aquatiques (FAO, 2014), cette dépendance accrue occasionne à cet effet une pression très forte sur les pêcheries par un effort de pêche incontrôlé, une utilisation généralisée de pratiques et d'engins de pêche non sélectifs et surtout, par l'absence d'une politique soutenue d'orientation des pêcheurs vers les ressources halieutiques encore inexploitées ou sousexploitées et vers les activités alternatives. Du fait de la pression démographique, des insuccès scolaires et de la misère grandissante au sein des populations locales, on assiste à une pression de pêche sur les stocks de poissons avec comme conséquence l'amenuisement des captures débarquées dans les pêcheries (Mukabo et al., 2017). La composition spécifique des captures s'est considérablement modifiée au fil des années et se caractérise de nos jours par une forte domination des espèces de petites tailles (Lalèyè et al., 2007). Avec cette pression démographique, la pêche sur les différents plans d'eau n'arrive plus à permettre aux pêcheurs de subvenir à leurs besoins. C'est la même situation au niveau du lac Toho qui s'est appauvri considérablement en ressources halieutiques passant de 45310 à 37723 tonnes de 2014 à 2016 (FAO, 2018 ; INSAE, 2018). Des études effectuées sur certaines pêcheries du Bénin ont montré que l'utilisation massive d'engins et techniques de pêche traditionnels et rudimentaires (Lalayè et al., 2005), les nasses et les acadjas (Attingli et al., 2017) sont à la base de cette baisse de ressources halieutiques. Le niveau de la dégradation continue de cet écosystème engendre, de fait, une extrême pauvreté des populations riveraines (Anticamara et al., 2011). L'objectif de cet article est d'analyser les déterminants de l'utilisation des pratiques de pêche destructives des ressources halieutiques dans lac Toho. 


\section{MATERIEL ET METHODES \\ Zone d'étude}

L'étude a été réalisée dans les villages Vèha, Logbo (commune de Lokossa), Tohonou et Tokpa (commune de Houéyogbé) qui sont des villages se situant au bord du lac Toho (Figure 1). Le lac Toho $\left(6^{\circ} 3635-6^{\circ} 40 \mathrm{~N}\right.$ et $\left.1^{\circ} 45-1^{\circ} 50 \mathrm{E}\right)$ est situé au sud du Bénin. Sa surface varie entre $9,6 \mathrm{~km}^{2}$ et $15 \mathrm{~km}^{2}$ avec une profondeur moyenne de 2,1 m (Ahouansou et al., 2008). Il a en moyenne $7 \mathrm{~km}$ de longueur ; de largeur méridionale variant entre 0,5 et 2,5 $\mathrm{km}$ et environ $500 \mathrm{~m}$ de largeur septentrionale. La vallée de la rivière Sazué sert de débouché pendant la saison des crues à travers deux canaux. Cette vallée sert également d'affluent lors des inondations du Mono. En raison de sa situation géographique, le lac Toho est influencé par un climat subéquatorial caractérisé par quatre saisons distinctes: (1) une longue saison des pluies de mi-mars à mijuillet, (2) une saison sèche de mi-juillet à miseptembre, (3) une courte saison des pluies de mi-septembre à mi-novembre et (4) une longue saison sèche de mi-novembre à mi-mars dominée par les vents continentaux et l'harmattan (Lederoun et al., 2015). Les précipitations annuelles varient entre $544 \mathrm{~mm}$ et $1376 \mathrm{~mm}$ tandis que la température se situe entre 20,6 et $33,5{ }^{\circ} \mathrm{C}$ avec une moyenne annuelle de $28{ }^{\circ} \mathrm{C}$. L'humidité relative est très élevée et varie de $65 \%$ en janvier à $80,6 \%$ en juin (Ahouansou et al., 2008).

\section{Collecte des données}

Les données ont été collectées en deux phases complémentaires, la phase exploratoire et la phase d'enquête approfondie. La phase exploratoire a permis d'identifier les villages impliqués dans les activités de pêche sur le lac Toho. La phase d'enquête approfondie est constituée de l'échantillonnage et de la collecte des données primaires. L'échantillonnage a consisté à la sélection des villages, au recensement de tous les ménages pêcheurs dans chaque village retenu, et à la sélection aléatoire des ménages pêcheurs dans lesdits villages. La sélection des villages a tenu compte, entre autres, de l'activité dominante, du poids démographique de chaque village et de la position géographique par rapport au lac. Ainsi, quatre villages ont été sélectionnés : Vèha et Logbo dans la commune de Lokossa et Tokpa et Tohonou dans la commune de Houéyogbé. Le recensement des ménages ayant d'impact sur la ressource a donné comme résultat 241 ménages répartis comme suit : 43 ménages pêcheurs à Vèha, 45 à Logbo, 93 à Tokpa et 60 à Tohonou. Pour déterminer la taille de l'échantillon correspondant à cette population, la méthode PPI (Progress out of Poverty Index) a été utilisée. Avec cette méthode, la taille de l'échantillon (n) des ménages à enquêter est fixée avec un niveau de confiance (1-c) $\%=95 \%$. La formule de taille $\mathrm{n}$ d'échantillon définie dans le cadre du Progress out of Poverty Index est

$$
n=N * \frac{z^{2} * \alpha^{2} * p(1-p)}{z^{2} * \alpha^{2} * p(1-p)+c^{2} *(N-1)}
$$

(Schreiner, 2012)

$$
c= \pm Z * \alpha * \sqrt{\frac{p(1-p)}{n}} * \sqrt{\frac{(N-n)}{N-1}}
$$

Avec:

$\checkmark \mathrm{N}$ est la taille de la population totale.

$\checkmark \mathrm{z}=1,96$, représente la valeur de la variable aléatoire normale $U$ pour un intervalle de confiance égal à 0,05 ;

$\checkmark \mathrm{c}$ (Intervalle de confiance) $=0,05$;

$\checkmark$ p (le pourcentage de la population qui pratique la pêche dans la zone d'étude, $\hat{p}=$ $67 \%)$;

$\checkmark \alpha$ (Les intervalles de confiance pour le PPI $=0,90)$;

Ainsi pour $\mathbf{N}=\mathbf{2 4 1}$, on a $\mathbf{n}=\mathbf{1 2 9}$.

Pour trouver l'effectif des souséchantillons dans chaque village retenu, il a été utilisé à cet effet la méthode de proportionnalité. Pour ce faire, un coefficient $\mathrm{k}$, appelé taux de sondage ou d'échantillonnage a été calculé, tel que : $\mathrm{k}=\frac{\mathrm{n}}{\mathrm{N}}(53 \%)$. Avec ce taux, les échantillons par village sont : 23 (Vèha), 23 (Logbo), 50 (Tokpa) et 33 (Tohonou). La collecte des données primaires est faite à l'aide d'un questionnaire administré aux chefs de ménage pêcheurs. 


\section{Méthodes d'analyse des données \\ Modèle théorique d'analyse}

Pour identifier les facteurs déterminants de l'utilisation des engins et pratiques de pêche destructives des ressources halieutiques, le modèle économétrique Logit binomial a été utilisé. Le Logit est fondé sur la loi logistique. La variable dépendante a deux modalités : soit l'enquêté utilise au moins une pratique destructive de pêche soit il n'utilise pas. En se référant à Peng et al. (2002), Agresti et al. (2009), et Rakotomalala (2015), l'équation du modèle se présente sous la forme suivante :

$\ln \left(\frac{\pi}{1-\pi}\right)=\alpha+\beta X(1)$

La linéarisation de l'équation

donne l'équation (2):

$\pi=P(Y)=\frac{e^{\alpha+\beta X_{i}}}{1+e^{\alpha+\beta X_{i}}}(2)$

Où $\pi$ est la probabilité que le pêcheur utilise au moins une pratique destructive de pêche (Y $=1$ ), $\mathrm{Xi}=$ vecteur des variables explicatives, $\alpha$ est la constante de l'équation, $\beta$ représentent les coefficients de régression des variables explicatives à estimer.

La variable dépendante $\mathrm{Yi}$ $=\left\{\begin{array}{c}\mathbf{1} \text { si le pêcheur emploie au moins une } \\ \text { pratique destructive de pêche } \\ \mathbf{0} \text { sinon }\end{array}\right.$

En prenant le log de l'équation (2), nous obtenons le modèle Logit suivant avec les coefficients à estimer :

$\ln \left(\frac{\mathrm{P}(\mathrm{Y}=1)}{1-\mathrm{P}(\mathrm{Y}=1)}\right)=\alpha+\beta_{1} \mathrm{X}_{1}+\beta_{2} \mathrm{X}_{2}+$

$\cdots \beta_{\mathrm{k}} \mathrm{X}_{\mathrm{k}}+\mathrm{e}_{\mathrm{i}}(3)$

$e_{i}$ sont les termes d'erreurs et $\mathrm{i}=1,2,3,4, \ldots . \mathrm{k}$ sont les observations.

Ensuite, on estime l'équation (3) par l'estimation des coefficients $\alpha, \beta_{1}, \beta_{2} \ldots \beta_{k}$ et enfin l'interprétation des résultats du modèle.

\section{Modèle empirique}

La probabilité que le pêcheur utilise au moins une pratique destructive de pêche peut s'écrire (Rakotomalala, 2015):

$$
\begin{aligned}
\ln \left(\frac{\mathrm{P}(\mathrm{Y}=1)}{1-\mathrm{P}(\mathrm{Y}=1)}\right) & =\alpha+\beta_{1} \text { EXP }+\beta_{2} \text { ACTPRIN } \\
& +\beta_{3} \text { FREQ } 1+\beta_{4} \text { FREQ } 2 \\
& +\beta_{5} \text { NIV_1 }+\beta_{6} \text { NIV_2 }
\end{aligned}
$$

Le logiciel STATA 13 a été utilisé pour analyser les données. La validation du modèle logit binomiale s'est faite avec le ratio de vraisemblance dont la probabilité doit être inférieure au seuil de $5 \%$. Le pseudo- $\mathrm{R}^{2}$ de Mcfadden donne le pourcentage de la variation de la variable dépendante par les variables explicatives introduites dans le modèle Logit binomiale. Les variables retenues à cet effet dans le modèle sont celles qui ont été significatives au seuil de $1 \%, 5 \%$ ou $10 \%$.

Le Tableau 1 présente la définition des variables explicatives du modèle et leurs signes attendus.

AGE : représente l'âge du pêcheur. L'âge est une variable socio-économique qui influencera de façon négative l'utilisation des techniques et pratiques destructives de pêche. Plusieurs travaux ont utilisé cette variable dans les modèles économétriques tels que Allison et al. (2001) et Daw et al. (2012).

EXP : représente le nombre d'années d'expériences du pêcheur au cours desquelles il a mobilisé plusieurs connaissances endogènes qu'il utilise pour apprécier, analyser et interpréter les faits et phénomènes liés à la pêche. Le nombre d'années d'expériences est un facteur qui sera corrélé de façon négative avec l'utilisation des pratiques destructives de pêche en ce sens où plus le nombre d'années d'expériences dans la pratique de la pêche augmente, plus le pêcheur prendra conscience $\mathrm{du}$ problème (baisse des ressources halieutiques) et développera des stratégies ou mesures allant dans le sens de la résolution dudit problème. Cette variable a été utilisée par Pollnac et al. (2001), Pita et al. (2010) et Daw et al. (2012).

ATPRIN : représente l'activité principale de l'enquêté, l'activité dans laquelle l'individu se reconnaît et y consacre beaucoup de temps. Le pêcheur et son ménage dépendent en grande partie de cette activité principale. Il investit la plupart de ses moyens dans cette activité pour avoir le maximum de satisfaction. Ce qui peut à l'amener à utiliser tous les moyens nécessaires y compris les pratiques destructives pour pêcher le maximum de ressources halieutiques. L'activité principale (pêche) du pêcheur influencerait de façon positive l'utilisation des pratiques destructives de pêche. Cette variable a été utilisée par Allison et al. (2001), Marshall et al. (2007) et 
Daw et al. (2012) dans leurs modèles économétriques.

FREQ : La fréquence de pêche est un facteur capital pour analyser la baisse des ressources halieutiques. C'est un facteur qui va influencer de façon positive l'utilisation des pratiques destructives de pêche. En effet, le pêcheur fréquente le lac en fonction de ses engins et techniques de pêche. Un pêcheur qui possède un engin de pêche pouvant capturer toute catégorie d'âge de poissons (filets maillants par exemple), aura tendance à plus fréquenter le lac afin d'augmenter à chaque fois les prises. Plusieurs études (Coulthard, 2008 ; Daw et al., 2012) ont utilisé cette dans l'analyse des déterminants socio-économiques.
NIV : désigne le niveau d'instruction de l'enquêté. L'éducation contribue par l'introduction de nouvelles valeurs, à la transformation des modèles culturels et traditionnels. En effet, l'instruction permet à l'individu de réaliser des progrès au niveau de sa vie. Un niveau d'instruction élevé peut permettre à l'enquêté d'avoir une grande connaissance formelle sur les ressources halieutiques et des stratégies de gestion durable de ces ressources. Le niveau d'instruction du pêcheur est une variable qui sera corrélée de façon négative avec l'utilisation des pratiques destructives de pêche. Cette variable a été utilisée par Allison et al. (2001), Marshall et al. (2007), Smith et al. (2005) et Daw et al. (2012).

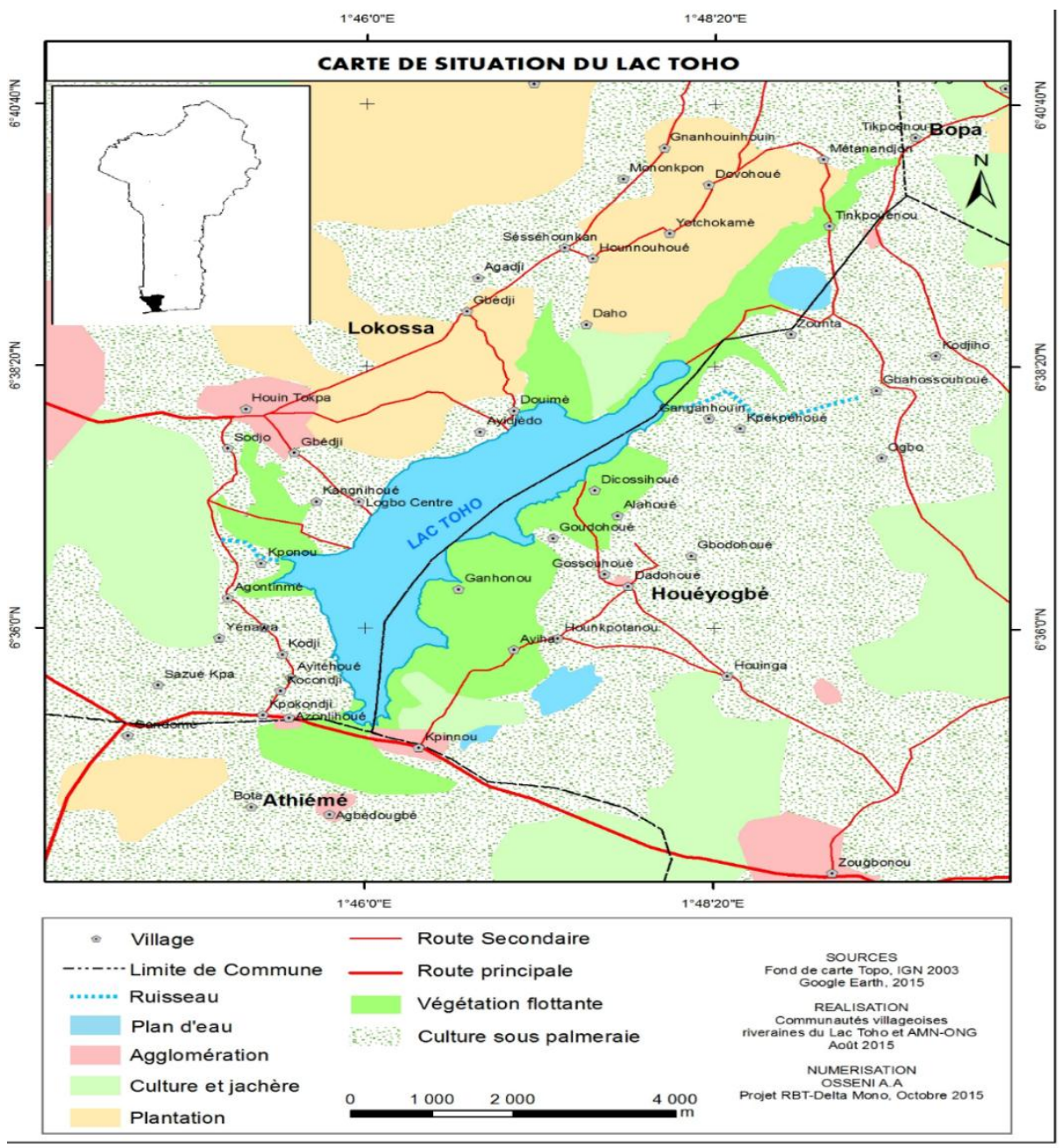

Figure 1 : Localisation de la zone d'étude.

Source : Fond de carte Topo, IGN 2003. Google Earth, 2015 
Tableau 1 : Définition des variables du modèle Logit et signes attendus.

\begin{tabular}{|c|c|c|c|}
\hline Variables & Code & Modalités & Signe attendu \\
\hline Age du pêcheur & AGE & Continue & - \\
\hline Nombre d'années d'expérience & EXP & Continue & - \\
\hline Activité principale & ACTPRIN & $\begin{array}{l}1: \text { Pêche ; 0 : Autres (Agriculture, } \\
\text { Elevage, Chasse, Transformation) }\end{array}$ & + \\
\hline Fréquence de pêche 1 & FREQ_1 & $\mathbf{1}:$ Une fois par jour $; \mathbf{0}:$ Sinon & + \\
\hline Fréquence de pêche 2 & FREQ_2 & $\begin{array}{l}1 \text { : Plus d'une fois par jour ; } 0 \text { : } \\
\text { Sinon }\end{array}$ & + \\
\hline Niveau d'instruction primaire & NIV_1 & $\mathbf{1}:$ Primaire $; \mathbf{0}:$ Sinon & - \\
\hline $\begin{array}{ll}\text { Niveau } & \text { d'instruction } \\
\text { secondaire } & \end{array}$ & NIV_2 & 1 : Secondaire ; 0 : Sinon & - \\
\hline
\end{tabular}

\section{RESULTATS}

\section{Caractéristiques socio-économiques des pêcheurs}

Le Tableau 2 relatif aux caractéristiques socio-économiques montre la répartition des pêcheurs suivant leur âge, le nombre d'années d'expérience dans la pêche (première partie du Tableau 2). Les pêcheurs ont en moyenne $40,35( \pm 10,18)$ ans. Ils ont en moyenne 38,17 $( \pm 9,15)$ années d'expériences dans la pratique de pêche. La seconde partie du tableau 2 relative aux variables qualitatives a montré que la majorité des pêcheurs $(69,18 \%)$ ont la pêche comme activité principale. $53,49 \%$ et $85,18 \%$ respectivement des pêcheurs n'ont aucun niveau d'instruction et fréquentent habituellement le lac Toho plus d'une fois par jour.

\section{Perceptions sur les pratiques destructives de pêche}

Les pratiques destructives de pêche (Figure 2) expliquant la baisse des ressources halieutiques sont respectivement par ordre d'importance la pratique de la pêche à la main (82,3\%), l'utilisation des épuisettes $(71,1 \%)$, l'utilisation des filets et nasses à petites mailles (mailles inférieures à $40 \mathrm{mn}$ ) $(58,7 \%)$ et l'utilisation des engrais chimiques utilisés lors de la production végétale au bord du lac $(15,1 \%)$. Les Figures 3, 4, 5 et 6 montrent respectivement les épuisettes, les filets maillants, les filets moustiquaires et le panier
«Gôdô» utilisés au cours de la pêche artisanale sur le lac Toho.

\section{Facteurs déterminants de l'emploi des} pratiques destructives de pêche

Le modèle Logit binomial réalisé pour déterminer les facteurs explicatifs de l'emploi des pratiques destructives de pêche donne les résultats qui sont présentés dans le Tableau 3. Le ratio de vraisemblance est significatif au seuil de $5 \%$ avec le test de khi-deux. Le pseudo- $\mathrm{R}^{2}$ de McFadden est 0,7915 et signifie que la variation des variables explicatives incluses dans le modèle explique à $79,15 \%$ de la variation de la variable dépendante qu'est l'utilisation des pratiques destructives de pêche. Par conséquent, le modèle est globalement significatif à $1 \%$ (Tableau 3). Le modèle a un pouvoir de prédiction correcte très élevé (supérieure à $90 \%$ ) et signifie que le modèle peut être utilisé pour prédire à $94,53 \%$ la variation de la variable dépendante suite aux variations des variables explicatives. Les variables significatives du modèle sont la fréquence de pêche 1 , la fréquence de pêche 2 , le nombre d'années d'expérience dans la pêche et le niveau d'instruction secondaire du pêcheur.

La variable "Nombre d'années d'expériences dans la pratique de la pêche" a un coefficient hautement significatif au seuil de $1 \%$ et de signe négatif (Tableau 3). Ce qui traduit que le "Nombre d'années d'expériences dans la pratique de la pêche" a un effet négatif 
sur la probabilité que le pêcheur utilise les pratiques destructives de pêche. Ce résultat satisfait donc le signe attendu du coefficient de la variable. L'effet marginal trouvé est significatif et de signe négatif.

La variable "Niveau d'instruction secondaire" du pêcheur a un coefficient significatif au seuil de $10 \%$ et de signe négatif. L'effet marginal de cette variable est aussi négatif. Ce qui traduit que le "Niveau d'instruction du pêcheur" a un effet négatif sur la probabilité que le pêcheur utilise les pratiques destructives de pêche. Ainsi, plus le niveau d'instruction du pêcheur évolue (vers le niveau secondaire), moins il emploie les pratiques destructives de pêche.

Les deux variables "Fréquence de pêche 1 " et "Fréquence de pêche 2 " ont des coefficients très hautement significatifs au seuil de $1 \%$ et de signe positif comme attendu. Les effets marginaux de ces deux variables étant positifs alors les pêcheurs qui fréquentent le lac une fois par jour d'une part et plus d'une fois par jour d'autre part emploient plus les pratiques destructives de pêche et occasionnent ainsi la baisse croissante des ressources halieutiques.

Tableau 2 : Caractéristiques socio-économiques des pêcheurs.

\begin{tabular}{|c|c|c|c|}
\hline \multicolumn{2}{|c|}{ Variables quantitatives } & Moyenne & Ecart type \\
\hline \multicolumn{2}{|c|}{ Age (années) } & 40,35 & 10,18 \\
\hline \multicolumn{2}{|c|}{ Nombre d'années d'expérience dans la pêche (années) } & 38,17 & 9,15 \\
\hline \multicolumn{3}{|c|}{ Variables qualitatives } & Pourcentage (\%) \\
\hline \multirow[t]{2}{*}{ Activité principale } & \multicolumn{2}{|l|}{ Pêche } & 69,18 \\
\hline & \multicolumn{2}{|c|}{ Agriculture, Elevage, Chasse, Transformation } & 30,82 \\
\hline \multirow[t]{3}{*}{ Niveau d'instruction } & Aucun & & 53,49 \\
\hline & Primaire & & 27,91 \\
\hline & Secondaire & & 18,60 \\
\hline \multirow[t]{2}{*}{ Fréquence de pêche } & Une fois par jour & & 14,88 \\
\hline & 2 à 5 fois par jour & & 85,18 \\
\hline
\end{tabular}

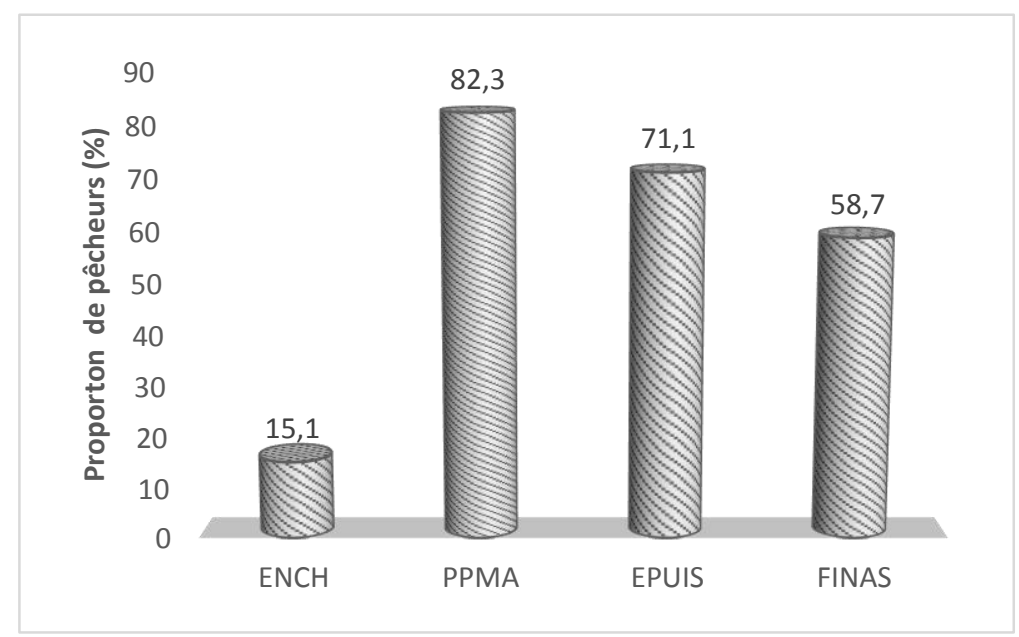

Figure 2 : Pratiques destructives de pêche au niveau du lac Toho.

Légende : ENCH : Engrais chimiques utilisés lors de la production végétale au bord du lac ; PPMA : Pratique de la pêche à la main ; EPUIS : Utilisation des épuisettes ; FINAS : Utilisation des filets et nasses à mailles serrées 


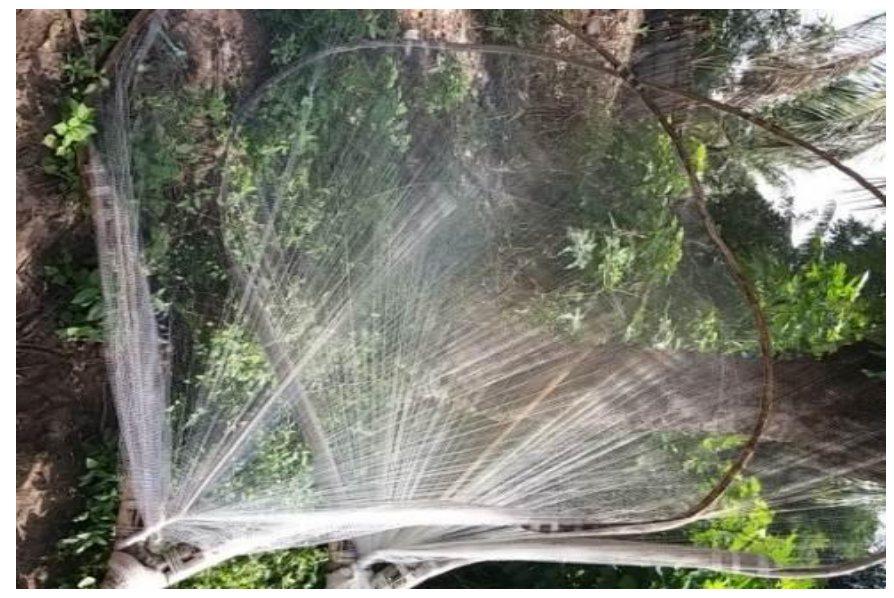

Cliché Codjo Victor, Lac Toho, 2016

Figure 3 : Grande épuisette utilisée au cours de la pêche sur le lac Toho.

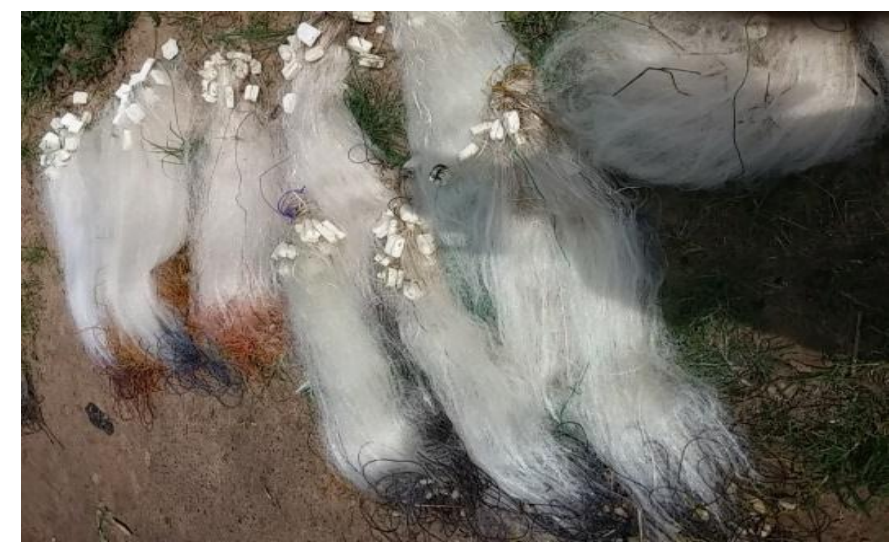

Cliché Codjo Victor, Lac Toho, 2016

Figure 4 : Filets maillants utilisés au cours de la pêche sur le lac Toho.

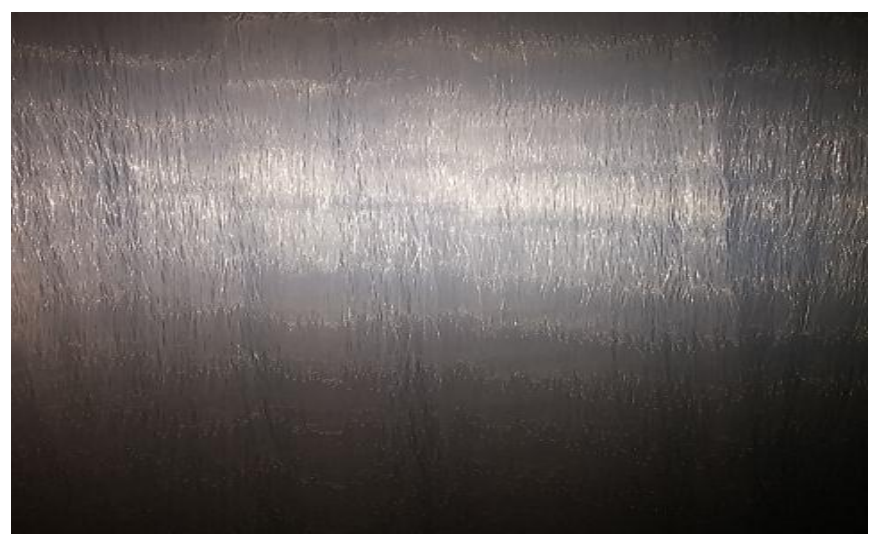

Cliché Codjo Victor, Lac Toho, 2016

Figure 5 : Filets moustiquaires utilisés au cours de la pêche sur le lac Toho. 


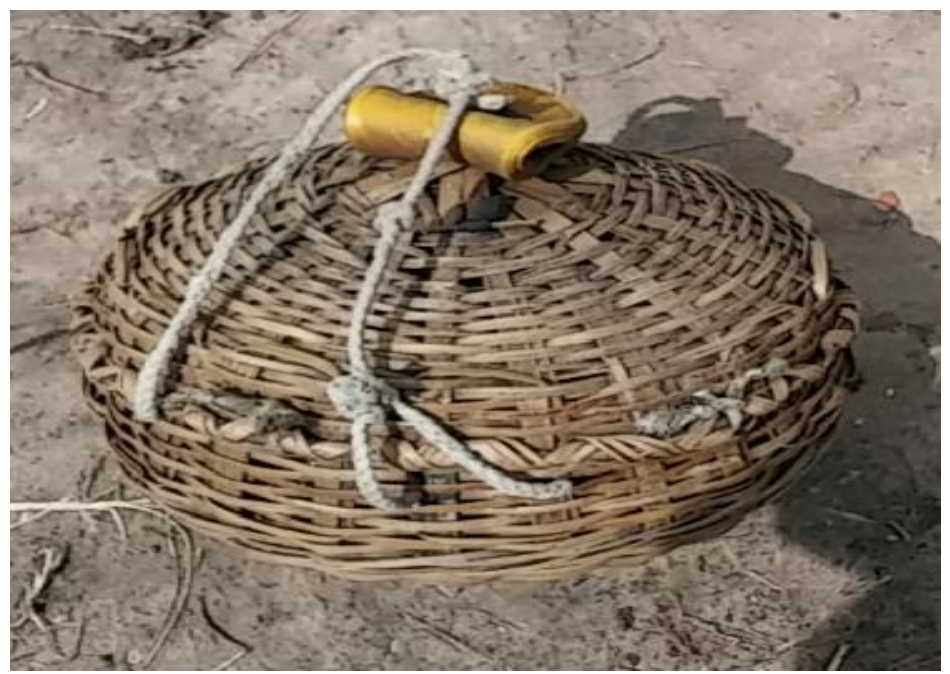

Cliché Codjo Victor, Lac Toho, 2016

Figure 6 : Panier «Gôdô » utilisé au cours de la pêche à la main.

Tableau 3 : Résultats du modèle logistique binomial.

\begin{tabular}{lccc}
\hline Variables & Coefficients & Erreur standard & Effets marginaux \\
\hline Age du pêcheur & $-0,56987$ & 0,568914 & 0,0078124 \\
Nombre d'années d'expériences & $-0,4429264^{* * * *}$ & 0,142206 & $-0,0026654^{* * *}$ \\
Activité principale & $-0,1756472$ & 1,618352 & $-0,001023$ \\
Fréquence de pêche 1 (Une fois par jour) & $0,385888^{* * *}$ & 0,1354023 & $0,0023222^{* * *}$ \\
Fréquence de pêche 2 (2 à 5 fois par jour) & $6,031985^{* * *}$ & 1,696446 & $0,3266^{* * *}$ \\
Niveau d'instruction primaire & 1,91145 & 1,59544 & 0,025154 \\
Niveau d'instruction secondaire & $-2,964446^{*}$ & 1,585857 & $-0,0270647^{*}$ \\
Constante & $-5,241864$ & 2,742724 & \\
\hline
\end{tabular}

Nombre d'observations $=129$

Signification du modèle (Prob > chi2) $=0,0000 * * *$

Log vraisemblance $=-13,472501$

Chi-deux (4) = 102,26

Pseudo $\mathrm{R}^{2}$ de McFadden $=0,7915$

$\%$ de prédiction correcte $=94,53 \%$

***= significatif à $1 \%$, ** = significatif à $5 \%$; * = significatif à $10 \%$. 


\section{DISCUSSION}

\section{Pratiques destructives de pêche et problème de baisse des ressources halieutiques}

Deux formes d'épuisettes (petites et grandes) sont utilisées sur le lac Toho (Ahouansou, 2003; Ahouansou et al., 2016). L'un des facteurs qui conduisent à la dégradation des stocks de poissons est le mode d'exploitation de ces ressources à travers les engins et techniques de pêche inadaptés (Lalayè et al., 2005 ; Akonkwa et al., 2017). Ainsi, selon Montchowui et al. (2007) et Attingli et al. (2017), les engins de pêche tels que "Acadja" et filets maillants sont les plus utilisés dans les zones de pêcherie situées en aval et au centre de la basse vallée de l'Ouémé (TU: 0,350 à 0,502) et ont un indice de nuisance élevé (IN: 0,209 chacun) sur l'ichtyofaune. L'influence des engins et techniques de pêches (II: 0,126 à 0,146 ) affecte aussi l'abondance relative des espèces de poissons (AR: 2,410 à 3,723). D'autres études effectuées sur le fleuve Ouémé (Montchowui et al., 2008) révèle que quatre types d'engins de pêche sont utilisés pour l'exploitation des poissons dans la vallée du fleuve Ouémé: le filet maillant, le filet épervier, la petite senne ou Akpélou et les nasses. Cette étude révèle un mode d'exploitation très sévère des poissons avec une forte pression de la pêche sur les juvéniles $0+$ issus de la reproduction de l'année et les poissons immatures; ce qui met en péril le bon renouvellement des poissons et favoriserait ainsi une dégradation de leur stock. Anticamara et al. (2016), dans la même logique, souligne que les filets éperviers et maillants permettent de capturer plus de poissons que les autres techniques de pêche et peuvent entrainer une diminution des ressources halieutiques. Selon Azaguagh et al. (2018), les différents engins de pêche (filet épervier, filet maillant, nasse et palangre) utilisés sur les lacs participent en grande partie aux prises totales. Ces filets maillants sont les engins de pêche les plus utilisés de manière permanente quelles que soient les saisons. Anticamara et al. (2016) soulignent que les engins de pêche les plus utilisés sur les lacs sont les filets maillants (filets ayant des mailles inférieures à $3 \mathrm{~mm}$ ) qui prélèvent une importante quantité de poissons. Les études empiriques menées dans le lac Tanganyika font ressortir que la pêche avec des engins destructifs est une importante menace à la biodiversité et au maintien des stocks exploités. C'est le cas des filets à trop petites mailles utilisés dans les frayères et nurseries, ce qui est particulièrement destructif pour les stocks de poissons, car tout est capturé, y compris les larves, les alevins, etc. Ces filets sont particulièrement dangereux parce qu'ils raclent le fond, retournent le substrat, obstruant ainsi les sources de nourriture et les nids des poissons (Mukabo et al. 2017). Par contre sur le Lac Mai-Ndombe au Congo, les trous à poissons, fréquemment utilisés par les pêcheurs artisans, constituent la technique de pêche destructive des ressources halieutiques (Luhusu et al., 2013). L'emploi des pratiques destructives de pêche (pratique de la pêche à la main et l'utilisation des filets éperviers et maillants) sur les ressources halieutiques des lacs est un problème reconnu puisque les prélèvements sur la ressource ont dépassé les capacités de renouvellement des stocks, ce qui est synonyme de surexploitation qui a conduit à une réduction de la biodiversité voire à l'extinction des ressources halieutiques (Badahoui et al., 2010 ; Dialla et al., 2016). La dégradation des ressources halieutiques au niveau des lacs est aussi causée par la pêche à la main (Machado et al., 2019). En effet, la pêche à la main est une technique qui consiste à aller au fond du lac et y rester pendant 5 à 10 minutes selon le pêcheur. Elle se fait le plus souvent en groupe. Lorsque les pêcheurs y sont, ils forment un cercle et utilisent leurs mains pour rechercher dans la boue les trous de poissons. Lorsque ces derniers arrivent à être repérés, les pêcheurs essayent de faire fuir ces poissons en détruisant ces trous. Au cours de leur fuite, ces poissons sont attrapés par les mains des pêcheurs qui avaient préalablement formé le cercle. Cette pratique est une pratique destructive des ressources halieutiques puisqu'elle entraîne non seulement la destruction des niches écologiques des poissons. C'est une technique de pêche qui compromet la reproduction et le renouvellement des espèces halieutiques. 


\section{Facteurs déterminants l'emploi des pratiques destructives de pêche}

Sur le lac Toho, les pêcheurs qui emploient plus les pratiques destructives de pêche sont ceux qui fréquentent plus le lac (une fois par jour ou soit plus d'une fois par jour), qui ont un faible nombre d'années d'expériences dans la pratique de la pêche et qui ont pour la plupart un niveau d'instruction primaire. La forte fréquentation des écosystèmes aquatiques à travers la multiplication du nombre de fois de pêche par jour ne favorise pas la reproduction et la croissance soutenue des poissons (Lalèyè et al., 2005 ; Akonkwa et al., 2017). Sur le Fleuve Ouémé au Bénin, cette forte fréquentation a entraîné une très forte pression de pêche sur les ressources halieutiques et provoque donc la baisse de la productivité de ces écosystèmes aquatiques (Lalèyè et al., 2007). Le nombre d'années d'expériences dans la pratique de la pêche est un facteur déterminant de l'emploi des pratiques destructives de pêche car plus le nombre d'années d'expériences dans la pratique de la pêche augmente, plus le pêcheur prend conscience de l'état actuel des ressources halieutiques et le compare à celui des années antérieures dans l'intention de prendre des mesures qui vont en faveur de la conservation des dites ressources. Ces mesures sont, entre autres : la réduction de l'emploi des pratiques destructives de pêche, l'utilisation des pratiques conservatrices des ressources halieutiques telles que les filets à mailles supérieures ou égales 50 millimètres (Montchowui et al., 2007 ; Ahouansou et al., 2016). Le respect de cette proportion de maille correspondant à la longueur optimale est une mesure de sauvegarde et de bonne gestion des ressources halieutiques telles que Sarotherodon galilaeus. Le "niveau d'instruction du pêcheur" est aussi un facteur déterminant dans l'appréciation de la baisse des ressources halieutiques. En effet, les pêcheurs n'ayant aucun niveau d'instruction et ceux ayant un niveau d'instruction primaire sont ceux qui utilisent plus, emploient plus les engins et pratiques de pêche destructives des ressources halieutiques tandis que ceux ayant un niveau d'instruction secondaire emploient plus des pratiques conservatrices des ressources halieutiques (Kpenavoun et al., 2017). Le constat est que la plupart des pêcheurs sont généralement des pauvres ayant un faible niveau d'instruction (Macusi et al., 2017 ; Machado et al., 2019). Quand le pêcheur accumule des connaissances formelles par l'éducation, il abandonne progressivement l'utilisation des pratiques destructives de pêche au détriment des pratiques conservatrices. Un niveau d'instruction élevé du pêcheur (niveau d'instruction secondaire) acquis grâce à l'éducation formelle induit, de fait, une prise de conscience sur l'état d'épuisement des ressources halieutiques du lac.

\section{Conclusion}

La présente étude sur l'analyse des déterminants socio-économiques de l'utilisation des pratiques de pêche destructives des ressources halieutiques du lac Toho au Bénin a mis en exergue non seulement la manière dont les pêcheurs perçoivent les pratiques destructives de pêche mais aussi les facteurs socio-économiques qui amènent les pêcheurs à employer ces pratiques. Elle a révélé que le nombre d'années d'expériences dans la pratique de la pêche, le niveau d'instruction du pêcheur et la fréquence de pêche du pêcheur sont donc les facteurs qui expliquent l'emploi des engins et pratiques de pêche destructives.

\section{CONFLITS D'INTERETS}

Les auteurs déclarent qu'ils n'ont aucun conflit d'intérêts pour cet article.

\section{CONTRIBUTIONS DES AUTEURS}

VC, AZ et GB ont tous travaillé sur la conception du manuscrit VC a spécialement a conçu les outils de collecte des données. Il a dirigé les travaux de saisie et de traitement des données collectées. L'analyse des données et la rédaction de l'article ont été faites en grande partie par VC. VC est le principal responsable de la rédaction de l'article. AZ et GB ont participé à l'analyse des données et à la rédaction de l'article. Ils sont les responsables de la qualité globale du document. 


\section{REMERCIEMENTS}

Les auteurs remercient la GIZ pour avoir permis la réalisation de l'étude exploratoire sur le Lac Toho dans le cadre du Projet Réserve de Biosphère Transfrontalière du Delta du Mono.

\section{REFERENCES}

Agresti A, Barbara F. 2009. Statistical Methods for the Social Sciences. Upper Saddle River, NJ: Pearson Hall Inc4th ed. p. 121.

Ahouansou MS, Lalèyè PA. 2008. Some aspects of biology of Oreochromis niloticus L (Perciformes: Cichlidae) recently introduced in Lake Toho (Benin, West Africa). Int. J. Biol. Chem. Sci., 2(1): 114-122. DOI : http://dx.doi.org/10.4314/ijbcs.v2i1.3972 9.

Ahouansou MS, Lonhodé JDS, Akotchéou AGG, Tokpanou, C F, Lederoun D, Lalèyè P. 2016. Détermination de maillage de filets pour une exploitation durable de Sarotherodon galilaeus à la retenue d'eau Gougan de Kogbétohoué au Bénin. Bulletin de la Recherche Agronomique du Bénin (BRAB), Numéro spécial Agronomie, Société, Environnement \& Sécurité Alimentaire : 85-92.

http://www.slire.net/download/2349/10_ me_article_brab_brab_n_sp_cial_projet_ niche-ben-174_-_ao_t_2016.pdf.

Ahouansou MS. 2003. Etude de l'écologie et de la production halieutique du lac Toho au Bénin. Mémoire de DESS, Faculté des Sciences Agronomiques, Université d'Abomey-Calavi, Bénin. p. 88.

Akonkwa B, Ahouansou MS, Nshombo M, Lalèyè P. 2017. Caractérisation de La Pêche au Lac Kivu. European Scientific Journal, $\quad$ 13(21): 269-292. DOI :

https://doi.org/10.19044/esj.2017.v13n21 p269.

Allison EH., Ellis F. 2001. The livelihoods approach and management of small-scale fisheries. Marine Policy, 25(5): 377-388.
DOI: $\quad$ https://doi.org/10.1016/S0308597X(01)00023-9.

Anticamara, JA, Watson RA, Gelchu, DP. 2011. Global fishing effort (1950-2010): trends, gaps, and implications. Fish. Res., 107: 131-136. DOI: https://doi.org/10.1016/j.fishres.2010.10. 016.

Anticamara JA, Go KTB. 2016. Spatiotemporal declines in Philippine fisheries and its implications to coastal municipal fishers' catch and income. Front. Mar. Sci. $\quad$ p. $13 . \quad$ DOI : https://doi.org/10.3389/fmars.2016.0002 1.

Attingli AH, Ahouansou MS, Vissin EW, Zinsou LH, Lalèyè PA. 2017. Influence des engins et techniques de pêche sur l'abondance relative des espèces de poissons dans la basse vallée de l'Ouémé au Bénin. African Crop Science Journal, 25(1) : $\quad 47-70 . \quad$ DOI : https://doi.org/10.4314/acsj.v25i1.4.

Azaguagh I, Driouchi A. 2018. Gestion des ressources halieutiques au Maroc et modes d'accès: Le modèle des «AntiCommons» et la pêcherie poulpière. Rev. Mar. Sci. Agron. Vét., 7(1): 5-17.

Badahoui A, Fiogbe ED, Boko M. 2010. Les causes de la dégradation du lac Ahémé et ses chenaux. Int. J. Biol. Chem. Sci., 4(4): 882-897.

DOI: https://doi.org/10.4314/ijbcs.v4i4.62971.

Coulthard S. 2008. Adapting to environmental change in artisanal fisheries Insights from a South Indian Lagoon. Global Environmental Change, 8: 479-489. DOI: http://doi:16/j.gloenvcha.2008.04.003.

Daw TM, Cinner JE, McClanahan TR., Brown K, Stead SM, Graham NAJ, Maina J. 2012. To Fish or Not to Fish: Factors at Multiple Scales Affecting Artisanal Fishers' Readiness to Exit a Declining Fishery. PLoS ONE, 7(2): 11. DOI : https://doi.org/10.1371/journal.pone.003 1460

Dialla Z, Tassembedo M, Micha JC. 2016. Mode d'exploitation et durabilité de la pêche de Oreochromis niloticus 
(Linnaueus, 1758), Clarias graiepinus (Burchell, 1822) et Gymnarchus niloticus (Cuvier, 1829) dans le lac de barrage du Sourou (Burkina Faso). Tropicultura, 34(4) : $\quad 350-360 . \quad$ DOI : http://www.tropicultura.org/index/author /dialla-z.html.

FAO. 2018. Situation mondiale des pêches et aquaculture : Contribuer à la sécurité alimentaire et à la nutrition de tous. Rome. 224 p.

FAO. 2014. Pêche et sylviculture. Fisheries and Aquaculture Department. Food and Agriculture Organization of the United Nations, Rome. 95 p.

Institut National de la Statistique et de l'Analyse Economique (INSAE). 2018. Evolution de la production halieutique de 1998 à 2018. https://www.insaebj.org/statistiques/statistiqueseconomiques/124-les-statistiquesagricoles .

Kpenavoun CS, Gandonou E, Adégbidi A, Abokini E. 2017. Mesure et déterminants de l'efficacité technique des pisciculteurs du Bénin. Int. J. Biol. Chem. Sci., 11(5): 2194-2208.

DOI: https://dx.doi.org/10.4314/ijbcs.v11i5.20

Lalèye P, Akele D, Philippart JC. 2007. La pêche traditionnelle dans les plaines inondables du fleuve Ouémé au Bénin. Cahiers d'Ethologie, 22(2): 25-38. DOI : http://hdl.handle.net/2268/27340.

Lalèyè $\mathrm{P}$, Salako $\mathrm{O}$, Chikou $\mathrm{A}$, Philippart JC. 2005. Artisanal gill-net fishery catches of the catfish, Schilbe intermedius (Teleostei: Schilbeidae) in two ributaries of Ouémé river, Bénin, West Africa. African Journal of Aquatic Science, 30(2): $\quad$ 163-166. DOI: https://dx.doi.org/10.2989/16085910509 503851.

Lederoun D, Chikou A, Vreven E, Snoeks J, Moreau J, Vandewalle P, Lalèyè P. 2015. Population parameters and exploitation rate of Sarotherodon melanotheron melanotheron rüppell, 1852 (Cichlidae) in Lake Toho, Benin. Journal of Biodiversity and Environmental Sciences (JBES), 6(2): 259-271.
Luhusu KF, Micha JC. 2013. Analyse des modes d'exploitation des ressources halieutiques du Lac Mai-Ndombe en République Démocratique du Congo. Geo-Eco-Trop., $\quad 37(2): \quad 273-284$. https://researchportal.unamur.be/fr/publi cations/analysis-of-exploitation-meansof-lake-mai-ndombe-fisheries-resou

Machado AM, da S, Daura-Jorge FG, Herbst $\mathrm{DF}$, Simões-Lopes PC, Ingram SN, Castilho PV, Peroni N. 2019. Artisanal fishers' perceptions of the ecosystem services derived from a dolphin-human cooperative fishing interaction in southern Brazil. Ocean \& Coastal Management, 173: 148-156. DOI: https://doi.org/10.1016/j.ocecoaman.201 9.03.003.

Macusi ED, Katikiro RE, Babaran RP. 2017. The influence of economic factors in the change of fishing strategies of anchored FAD fishers in the face of declining catch, General Santos City, Philippines. Marine Policy, 78: 98-106. DOI: https://doi.org/10.1016/j.marpol.2017.01. 016.

Marshall NA, Fenton DM, Marshall PA, Sutton SG. 2007. How Resource Dependency Can Influence Social Resilience within a Primary Resource. Industry. Rural Sociology, 72: 359-390. DOI: https://doi.org/10.1526/00360110778179 9254.

Ministère de l'Agriculture, de l'Elevage et de la Pêche (MAEP). 2009. Statistiques agricoles. Direction des Pêches. Cotonou, Bénin. p. 57.

Montchowui E, Niyonkuru C, Ahouansou MS, Chikou A, Lalèyè P. 2007. L'ichtyofaune de la rivière Hlan au Bénin (Afrique de l'Ouest). International journal of ichthyology, $31(2): \quad 5$. https://www.google.com/url?sa=t\&rct=j $\& \mathrm{q}=\&$ esrc $=\mathrm{s} \&$ source $=$ web $\& \mathrm{~cd}=\& \mathrm{cad}=\mathrm{rj}$ a\&uact $=8 \&$ ved $=2$ ahUKEwja34Ts $2 P P r A$ hXNzYUKHVo8CroQFjAAegQIAhAB \&url=http $\% 3 \mathrm{~A} \% 2 \mathrm{~F} \% 2$ Faquadico.com $\%$ 2Fup\%2FRiviere_Hlan_Benin_copie.pdf \&usg=AOvVaw0YYej076VWhsOhCFvCI5u. 
Montchowui E, Tobada P, Chikou A, Laleye P. 2008. Caractéristiques et impact de la pêche artisanale sur l'exploitation de Labeo senegalensis (Valenciennes, 1842) dans la basse vallée du fleuve Ouémé au Bénin. Int. J. Biol. Chem. Sci., 2(2) : 478489.

DOI : https://doi.org/10.4314/ijbcs.v2i4.39763.

Mukabo OG, Micha JC, Habarugira JB, Ntakimazi G, Nshombo MV, Bizuru NP, Muhirwa BG. 2017. Socio-économie de la pêche artisanale dans les eaux burundaises du lac Tanganyika à Mvugo et Muguruka. Int. J. Biol. Chem. Sci., 11(1): 247-265. DOI: http://dx.doi.org/10.4314/ijbcs.v11i1.20.

Peng CYJ, So TSH. 2002. Logistic Regression Analysis and Reporting: A Primer. Understanding Statistics, 1(1): 31-70. DOI:

https://doi.org/10.1207/S15328031US01 01_04.

Pita C, Dickey H, Pierce GJ, Mente E, Theodossiou I. 2010. Willingness for Mobility amongst European fishermen. Journal of Rural Studies, 26(3): 308-319. DOI:

http://dx.doi.org/10.1016/j.jrurstud.2010. 02.004 .

Pollnac RB, Pomeroy RS, Harkes IHT. 2001. Fishery policy and job satisfaction in three South East Asian fisheries. Ocean \& Coastal Management, 44(7-8): 531-544. DOI: https://doi.org/10.1016/S09645691(01)00064-3.
Rakotomalala R. 2015. Pratique de la Régression Logistique- Régression Logistique Binaire et Polytomique. Université Lumière Lyon 2. Version 2.0. $130 \mathrm{p}$.

Roche International. 2000. Etude du Projet d'aménagement des plans d'eau du SudBénin : Le secteur des pêches. Volume 3, Tome 3. p. 140.

Schreiner M. 2012. Progress out of Poverty Index (PPI): A Simple Poverty Scorecard for Benin. www.progressoutofpoverty.org. p. 2.

Smith LED, Khoa SN, Lorenzen K. 2005. Livelihood functions of inland fisheries: Policy implications for developing countries. Water Policy, 7(4): 359-383. DOI : https://doi.org/10.2166/wp.2005.0023.

Ticheler A. 2000. Conservation de la biodiversité des poissons dans les zones humides de l'Afrique de l'Ouest. Plan d'action Régional 2000 Wetlands International. p. 90.

Union Economique et Monétaire Ouest Africaine (UEMOA). 2002. Grandes orientations de la Politique Commune d'Amélioration de l'Environnement (PCAE). Informations complémentaires sur les pays. Annexe 2, Volume 3. 7 - 45. 